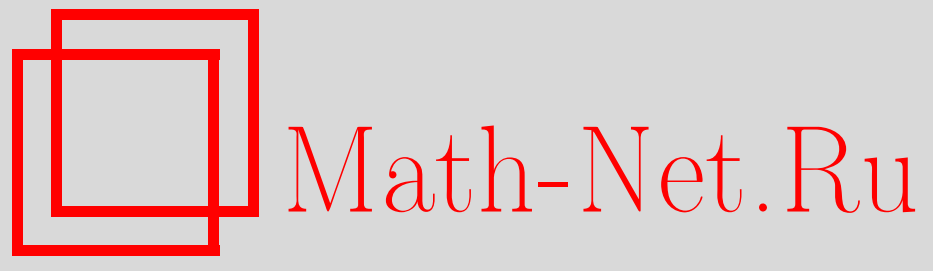

В. И. Ротарь, Об асимптотических разложениях для локальных структур зависимостей с сильным перемешиванием, Теория вероятн. и ее примен., 2007, том 52, выпуск 1, 21-40

DOI: https://doi.org/10.4213/tvp2

Использование Общероссийского математического портала MathNet.Ru подразумевает, что вы прочитали и согласны с пользовательским соглашением

http://www.mathnet.ru/rus/agreement

Параметры загрузки:

IP : 54.162 .127 .20

26 апреля 2023 г., 08:45:48

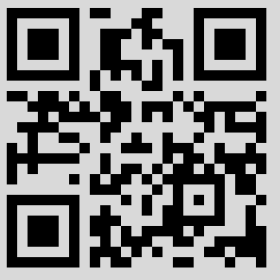




\title{
ОБ АСИМПТОТИЧЕСКИХ РАЗЛОЖЕНИЯХ ДЛЯ ЛОКАЛЬНЫХ СТРУКТУР ЗАВИСИМОСТЕЙ С СИЛЬНЫМ ПЕРЕМЕШИВАНИЕМ
}

\begin{abstract}
Эта статья развивает результаты, полученные в работе [28] и касающиеся разложения Эджворта для $\mathbf{E} h(W)$, где $W$ - сумма зависимых случайных величин и $h(x)$ - некоторая функция, для случая так называемых цепочек окрестностей зависимости. Наиболее известным примером таких структур является перемешивание на графах. Основное улучшение состоит в замене характеристик $\phi$-перемешивания характеристиками сильного (или $\alpha$-) перемешивания, что существенно расширяет возможности применения. Другое улучшение касается условий гладкости. Мы покажем, как условия гладкости $h$ могут быть заменены условиями гладкости распределения $W$.
\end{abstract}

Ключевые слова и фразы: разложение Эджворта, локальная зависимость, окрестность зависимости, метод Стейна.

\section{1. Введение и результаты}

1.1. Предыстория и мотивация. Пусть $h(\cdot)$ - некоторая функция и $W=X_{1}+\cdots+X_{n}$, где $X_{i}$ - случайные величины. Эта статья посвящена асимптотическим разложениям для $\mathbf{E} h(W)$.

Результаты, касающиеся асимптотических разложений для различных типов зависимости, и дальнейшие ссылки можно найти, например, в статьях (в хронологическом порядке) [33], [15], [24], [18] (цепи Маркова); [11], [21] (классическое перемешивание); [27] (m-зависимые случайные величины); [12]-[14] (m-зависимые случайные величины и случайные поля); [25] (мартингалы); [19] (случайные поля); [20], [6], [22], [23], [7], [3] (U-статистики); [4] (симметричные статистики). Надо заметить, что данный список далеко не полон.

В этой статье мы продолжаем изучать асимптотические разложения для $\mathbf{E} h(W)$; начало этой работы было положено в статье Й. Ринотта

* Department of Mathematics and Statistics of San Diego State University и Центральный экономико-математический институт РАН, Нахимовский пр-т, 47, 117418 Москва, Рослия; e-mail: vrotar@math.ucsd.edu 
и автора [28], где структуры зависимости были описаны в терминах разложений $W$.

В типичной ситуации указанные разложения определяются так называемыми окрестностями зависимости (dependency neighborhoods), когда для каждого слагаемого $X$ в сумме указывается множество слагаемых, от которых $X$ существенно зависит. Более точно, для каждого слагаемого $X$ мы рассматриваем цепочку множеств слагаемых $\mathscr{N}_{1}, \ldots, \mathscr{N}_{d}$ такую, что $X \in \mathscr{N}_{1} \subseteq \cdots \subseteq \mathscr{N}_{d}$. Мы предполагаем, что $X$ в определенном смысле существенно зависит только от величин из $\mathscr{N}_{1}$ и слабо - от величин, не принадлежащих $\mathscr{N}_{1}$. Аналогично, множество слагаемых $\mathscr{N}_{1}$ существенно зависит только от величин из $\mathscr{N}_{2}$, и слабо - от величин, не принадлежащих $\mathscr{N}_{2}$; и т.д. В случае независимых слагаемых мы определяем все множества $\mathscr{N}_{s}$ как содержащие только $X$.

Аналогичные разложения можно найти в [2], [29], а также в ссылках, приведенных в этих работах.

Существенная гибкость такой схемы достигалась благодаря, в частности, тому, что рассматриваемая в [28] и в настоящей статье последовательность $\mathscr{N}_{1}, \ldots, \mathscr{N}_{d}$ является произвольной и для каждой отдельной структуры зависимости можно определить такую последовательность $\left\{\mathscr{N}_{s}\right\}$, для которой ошибка аппроксимации была бы мала. В частности, окрестности $\mathscr{N}_{s}$ могут быть случайными, могут зависеть от значений $X$ и т.д.

Самым типичным примером применения является перемешивание на графах, т.е. когда параметр, индексирующий слагаемые, который обычно понимается как «время» или «пространство», принимает значения, которые можно идентифицировать с вершинами графа; см. [28].

Остаточный член в разложениях в [28] и в данной статье оценивается в терминах отношений Ляпунова или близких характеристик. Упомянутую выше слабую зависимость можно оценить с помощью условий, близких к условиям перемешивания.

В [28] в качестве меры зависимости между двумя $\sigma$-алгебрами $\mathscr{A}$ и $\mathscr{B}$ использовалась мера

$$
\phi(\mathscr{A}, \mathscr{B}):=\sup \{|\mathbf{P}\{B \mid A\}-\mathbf{P}(B)| ; A \in \mathscr{A}, B \in \mathscr{B}, \mathbf{P}(A)>0\} .
$$

Заметим, что впервые условия ф-перемешивания были рассмотрены B [16].

Основная цель этой статьи - получить результат, аналогичный результату, полученному в [28], но в терминах меры сильного перемешивания (strong mixing measure)

$$
\alpha(\mathscr{A}, \mathscr{B}):=\sup \{|\mathbf{P}(A B)-\mathbf{P}(A) \mathbf{P}(B)| ; A \in \mathscr{A}, B \in \mathscr{B}\} .
$$

Условия сильного перемешивания впервые были использованы в [32]. 
Очевидно, что условия зависимости в терминах $\alpha$-перемешивания слабее, чем в терминах меры (1.1.1), и переход от $\phi$-перемешивания к $\alpha$-перемешиванию существенно расширяет возможности применений.

В п. 1.2.3 мы рассмотрим простой, но характерный пример ситуации, когда распределение $W$ асимптотически нормально, но характеристики в терминах $\phi$ не работают так, как это происходит в случае сильного перемешивания.

Метод, примененный в [28] и этой статье, использует подход Стейна и основан, в частности, на некотором специальном представлении (см. п. 2.2), впервые изученном для независимых слагаемых в работе [1] и существенно развитом в [28] для случая зависимых слагаемых. Упомянутое выше представление улучшается и обсуждается в работе [30], включая вопрос о переходе от $\phi$ - к $\alpha$-перемешиванию. Цель данной статьи - непосредственно разложения.

Другое улучшение результатов из [28] касается условий гладкости. В [28] рассматривались два типа условий: либо функция $h$ предполагалась достаточно гладкой, либо распределение суммы $W$ должно было быть достаточно гладким. Однако в разложениях, приведенных в [28], второй тип условий хорошо работает только в случае локальной зависимости, т.е. когда $X$ не зависит от величин, не принадлежащих $\mathscr{N}_{1}$, множество $\mathscr{N}_{1}$ не зависит от величин не принадлежащих $\mathscr{N}_{2}$, и т.д.

В этой статье мы покажем, как можно использовать условия гладкости второго типа в общем случае. Полученная форма остаточного члена позволяет нам сделать это с помощью стандартного сглаживания; см. замечание в самом начале п. 2.4.

Что касается других аспектов, кроме перемешивания и условий гладкости, результаты статьи близки к результатам из [28]. Поэтому мы можем сократить изложение, отослав читателя к [28] и [30]. То же касается примеров, которые были рассмотрены в специальном разделе в [28]. С другой стороны, дальнейшее изложение самодостаточно, и нет нужды обрашаться к другим статьям, чтобы понять основные результаты работы.

Полные доказательства приведенных ниже теорем достаточно длинные и сложные, но многие их части идентичны доказательствам из [28] или [30]. Мы не будем их повторять, но приведем доказательства модифицированных результатов из [28].

1.2. Основная схема. Фиксируем целое $d \geqslant 2$ и предположим, что

$$
\mathbf{E} X_{i}=0 \quad \text { и } \quad \mathbf{E}\left|X_{i}\right|^{d+1}<\infty \quad \text { для всех } i=1, \ldots, n .
$$

Во всех разложениях $d-$ наивысший порядок моментов, входящих в основной член разложений. 
1.2.1. Функция $h$. Зафиксируем некоторую функцию $h$, положим $h_{\varepsilon+}(x):=\sup _{|y| \leqslant \varepsilon} h(x+y), h_{\varepsilon-}(x):=\inf _{|y| \leqslant \varepsilon} h(x+y)$ и предположим, что

$$
\begin{gathered}
\omega(\varepsilon ; h):=\sup _{z} \int\left(h_{\varepsilon+}(x+z)-h_{\varepsilon-}(x+z)\right)\left(1+|x|^{3 d}\right) \Phi(x) d x \longrightarrow 0, \\
\text { если } \varepsilon \rightarrow 0
\end{gathered}
$$

где $\Phi$ - стандартная нормальная мера. Если $h$ является индикатором интервала или если $h^{(1)}$, т.е. производная $h,-$ равномерно ограниченная функция, что является одним из возможных условий, приведенных ниже, то очевидно, что (1.2.1) выполнено.

1.2.2. Разложения. Рассмотрим одно слагаемое $X=X_{i}$. Далее мы часто в обозначениях будем опускать индекс $i$, когда это не будет приводить к недоразумениям. Для каждого $X$ введем $d$ разложений

$$
W=W_{k}+\widetilde{W}_{k}, \quad k=1, \ldots, d .
$$

Отметим, что разложения (1.2.2) играют роль свободного парамет$p a$. В качестве примера, можно рассматривать $W_{k}$ как частичную сумму слагаемых, т.е. $W_{k}=\sum_{X_{j} \in \mathscr{N}_{k}} X_{j}$, где $X \in \mathscr{N}_{1} \subseteq \mathscr{N}_{2} \subseteq \cdots$, как выше, но это не является необходимым.

Пусть $W_{0}=0$ и $U_{s}=W_{s}-W_{s-1}$ для $1 \leqslant s \leqslant d$. Тогда $W_{s}=$ $U_{1}+\cdots+U_{s}$, и $\widetilde{W}_{s}=U_{s+1}+\widetilde{W}_{s+1}$ для $s=1, \ldots, d-1$. (В предыдущем примере $U_{s}=\sum_{X_{j} \in \mathscr{N}_{s} \backslash \mathscr{N}_{s-1}} X_{j}$.) В случае независимых величин положим все $W_{k}$ равными $X$.

1.2.3. Характеристика структуры зависимости. Пусть $\mathscr{F}_{0}=$ $\sigma\{X\}-\sigma$-алгебра, порожденная $X$, и $\mathscr{F}_{s}=\sigma\left\{X, U_{1}, U_{2}, \ldots, U_{s}\right\}$. Положим $\widetilde{F}_{s}=\sigma\left\{U_{s+1}, \ldots, U_{d}, \widetilde{W}_{d}\right\}, s=0,1, \ldots, d-1 ; \widetilde{\mathscr{F}}_{d}=\sigma\left\{\widetilde{W}_{d}\right\}$. Таким образом, между $\mathscr{F}_{s}$ и $\widetilde{\mathscr{F}}_{s+1}$ существует «брешь» $U_{s+1}$.

Вспоминая, что $X=X_{i}$, будем писать $U_{k i}, \widetilde{W}_{s i}$ и $\mathscr{F}_{s i}$ вместо $U_{k}, \widetilde{W}_{s}$ и $\mathscr{F}_{s}$ соответственно.

Мы говорим о локальной зависимости, если $\mathscr{F}_{s i}$ и $\widetilde{\mathscr{F}}_{s+1, i}$ независимы для всех $s=1, \ldots, d-1$ и $i=1, \ldots, n$.

Используя определение (1.1.2), введем характеристику зависимости

$$
K=\sup _{s, i} \alpha\left(\mathscr{F}_{s i}, \widetilde{\mathscr{F}}_{s+1, i}\right) .
$$

В случае локальной зависимости $K=0$.

В [28] была использована более грубая характеристика, а именно,

$$
T=\sup _{s, i} \phi\left(\mathscr{F}_{s i}, \widetilde{\mathscr{F}}_{s+1, i}\right),
$$

где $\phi$ определено в (1.1.1). Далее мы рассмотрим простой пример, когда асимптотическая нормальность очевидна, хотя характеристика $T$ 
не является малой, а $K$ мало. Для этого нам необходимы следующие представления:

$$
\begin{aligned}
& \phi(\mathscr{A}, \mathscr{B})=\frac{1}{2}\left\{\sup _{\xi_{1} \in \mathscr{L}_{1}(\mathscr{A}), \xi_{2} \in \mathscr{L}_{\infty}(\mathscr{B})} \frac{\left|\mathbf{E}\left\{\xi_{1} \xi_{2}\right\}-\mathbf{E} \xi_{1} \mathbf{E} \xi_{2}\right|}{\left\|\xi_{1}\right\|_{1}\left\|\xi_{2}\right\|_{\infty}}\right\}, \\
& \alpha(\mathscr{A}, \mathscr{B})=\frac{1}{2}\left\{\sup _{\xi_{1} \in \mathscr{L}_{\infty}(\mathscr{A}), \xi_{2} \in \mathscr{L}_{\infty}(\mathscr{B})} \frac{\left|\mathbf{E}\left\{\xi_{1} \xi_{2}\right\}-\mathbf{E}\left\{\xi_{1}\right\} \mathbf{E} \xi_{2}\right|}{\left\|\xi_{1}\right\|_{\infty}\left\|\xi_{2}\right\|_{\infty}}\right\} ;
\end{aligned}
$$

см., например, [9]. Доказательство того, что правая часть (1.2.5) не превосходит левую часть, см. в [9] и [17, лемма 1.1.9]; выбирая в качестве случайных величин индикаторы, можно практически достичь равенства. То же касается и (1.2.6).

Далее, во-первых, если для некоторого множества $D$ и случайных величин $\xi_{1}$ и $\xi_{2}$

$$
\mathbf{E}\left\{\xi_{1} \xi_{2} \mid D\right\}=\mathbf{E}\left\{\xi_{1} \mid D\right\} \mathbf{E}\left\{\xi_{2} \mid D\right\}
$$

то, как легко проверить,

$$
\begin{aligned}
\mathbf{E}\left\{\xi_{1} \xi_{2}\right\}-\mathbf{E} \xi_{1} \mathbf{E} \xi_{2}=\mathbf{P}\left(D^{c}\right)[ & \mathbf{E}\left\{\xi_{1} \xi_{2} \mid D^{c}\right\}+\mathbf{E}\left\{\xi_{1} ; D\right\} \mathbf{E}\left\{\xi_{2} \mid D\right\} \\
& \left.-\mathbf{E}\left\{\xi_{2} ; D\right\} \mathbf{E}\left\{\xi_{1} \mid D^{c}\right\}-\mathbf{E} \xi_{1} \mathbf{E}\left\{\xi_{2} \mid D^{c}\right\}\right]
\end{aligned}
$$

где $\mathbf{P}(D), \mathbf{P}\left(D^{c}\right) \neq 0$.

Во-вторых, если $\xi_{1} \in \mathscr{L}_{\infty}(\mathscr{A}), \xi_{2} \in \mathscr{L}_{\infty}(\mathscr{B})$ и $(1.2 .7)$ выполняется, то, учитывая $(1.2 .8)$ и (1.2.6), получаем, что

$$
\begin{aligned}
2 \alpha(\mathscr{A}, \mathscr{B})= & \sup _{\xi_{1} \in \mathscr{L}_{\infty}(\mathscr{A}), \xi_{2} \in \mathscr{L}_{\infty}(\mathscr{B})} \frac{1}{\left\|\xi_{1}\right\|_{\infty}\left\|\xi_{2}\right\|_{\infty}} \mathbf{P}\left(D^{c}\right) \\
& \times \mid \mathbf{E}\left\{\xi_{1} \xi_{2} \mid D^{c}\right\}+\mathbf{E}\left\{\xi_{1} ; D\right\} \mathbf{E}\left\{\xi_{2} \mid D\right\}-\mathbf{E}\left\{\xi_{2} ; D\right\} \mathbf{E}\left\{\xi_{1} \mid D^{c}\right\} \\
& -\mathbf{E} \xi_{1} \mathbf{E}\left\{\xi_{2} \mid D^{c}\right\} \mid \leqslant 4 \mathbf{P}\left(D^{c}\right) .
\end{aligned}
$$

Рассмотрим теперь частный случай.

П р и м е р. Пусть $Z_{1}, Z_{2}, \ldots$ - независимые случайные величины, распределенные так же как случайная величина $Z$, причем $\mathbf{E} Z=0$, $\mathbf{E} Z^{2}=1$. Чтобы упростить дальнейшие вычисления, предположим, что $Z$ симметрична и отделена от нуля, скажем, $|Z|>\frac{1}{2}$. Пусть $\beta_{n} \rightarrow 0$ при $n \rightarrow \infty$ и для фиксированного $n$ случайная величина $\eta_{(n)}= \pm \beta_{n}$ с равными вероятностями. Рассмотрим некоторое множество $D_{n}$ такое, что $\mathbf{P}\left(D_{n}\right)=1-\beta_{n}$, и вектор

$$
\left(Y_{1 n}, \ldots, Y_{n n}\right)= \begin{cases}\left(Z_{1}, \ldots, Z_{n}\right) & \text { на множестве } D_{n} \\ & \left(\text { с вероятностью } 1-\beta_{n}\right) \\ \left(\eta_{(n)}, \ldots, \eta_{(n)}\right) & \text { на множестве } D_{n}^{c} \\ & \left(\text { с вероятностью } \beta_{n}\right)\end{cases}
$$


(мы опускаем формальности определения всех случайных величин на одном пространстве). Пусть $S_{n}=Y_{1 n}+\cdots+Y_{n n}$. Очевидно, что последовательность $\left(S_{n} / \sqrt{n}\right)$ асимптотически нормальна.

Интуитивно понятно, и мы убедимся в этом в п. 1.3, что в этом случае естественный выбор разложений $(1.2 .2)$ - это $W_{k}=W_{k i}=X_{i}$ для всех $k=1, \ldots, d, i=1, \ldots, n$. Тогда $U_{1 i}=X_{i}$.

Отметим, что $T \geqslant \phi\left(\mathscr{A}_{1}, \widetilde{\mathscr{A}_{2}}\right)$, где $\mathscr{A}_{1}=\sigma\left(Y_{1 n}\right)$ и $\widetilde{\mathscr{A}}_{2}=\sigma\left(Y_{2 n}\right)$.

Чтобы определить нижнюю границу для $\phi\left(\mathscr{A}_{1}, \widetilde{\mathscr{A}}_{2}\right)$, мы воспользуемся (1.2.5). Положим $\xi_{1}=1 / Y_{1 n}$ и $\xi_{2}=\operatorname{sign}\left(Y_{2 n}\right)$. Тогда, в силу симметрии и (1.2.8),

$$
\mathbf{E}\left\{\xi_{1} \xi_{2}\right\}-\mathbf{E} \xi_{1} \mathbf{E} \xi_{2}=\mathbf{P}\left(D_{n}^{c}\right) \mathbf{E}\left\{\xi_{1} \xi_{2} \mid D_{n}^{c}\right\}
$$

Так как $Y_{1 n}=Y_{2 n}=\eta_{(n)}$ на $D_{n}^{c}$, то

$$
\mathbf{E}\left\{\xi_{1} \xi_{2}\right\}-\mathbf{E} \xi_{1} \mathbf{E} \xi_{2}=\mathbf{P}\left(D_{n}^{c}\right) \mathbf{E}\left\{|\eta|^{-1} \mid D_{n}^{c}\right\}=\beta_{n}\left(\beta_{n}^{-1}\right)=1 .
$$

Кроме того, учитывая (1.2.5) и то, что $|Z|>\frac{1}{2}$, получаем:

$$
\phi\left(\mathscr{A}_{1}, \widetilde{\mathscr{A}}_{2}\right) \geqslant \frac{\left|\mathbf{E}\left\{\xi_{1} \xi_{2}\right\}-\mathbf{E} \xi_{1} \mathbf{E} \xi_{2}\right|}{\left\|\xi_{1}\right\|_{1}\left\|\xi_{2}\right\|_{\infty}}=\frac{1}{\mathbf{E}\left\{|Z|^{-1}\right\}\left(1-\beta_{n}\right)+\left(1 / \beta_{n}\right) \beta_{n}} \geqslant \frac{1}{3}
$$

С другой стороны, мера зависимости $\alpha(\cdot, \cdot)$ в этом случае будет работать, учитывая (1.2.9); в нашем примере, благодаря выбору разложения, характеристика

$$
K \leqslant 2 \mathbf{P}\left(D_{n}^{c}\right)=2 \beta_{n}
$$

т.е. мала. Последняя оценка, примененная к общей оценке из теоремы 1 , ведет к правильной скорости сходимости в центральной предельной теореме для достаточно малых $\beta_{n}$.

1.2.4. Обобщенные моменты и кумулянты. Пусть $\mu_{l i}=$ $\mathbf{E}\left\{\left(\left|X_{i}\right|+\left|U_{1 i}\right|+\cdots+\left|U_{d i}\right|\right)^{l}\right\}, z_{k i}=\sum_{l=2}^{k} \mu_{l i}$ и

$$
\bar{\mu}_{l}=\sum_{i=1}^{n} \mu_{l i}, \quad \bar{z}_{k}=\sum_{i=1}^{n} z_{k i} \text {. }
$$

Для того чтобы прояснить порядок величин в (1.2.10), предположим, что $W$ уже нормирована таким образом, что

$$
\left|X_{i}\right| \leqslant \frac{Y}{\sqrt{n}} \text { по распределению, }
$$

где $Y$ - положительная случайная величина с $(d+1)$ конечными моментами. (Для двух случайных величин $X$ и $Y$ скажем, что $X \leqslant Y$ по распределению, если $\mathbf{P}\{X>x\} \leqslant \mathbf{P}\{Y>x\}$ для любого $x$. ) Также предположим, что случайные величины $U_{i}$ являются суммами ограниченного числа случайных величин $X$. Тогда легко показать, что $\bar{\mu}_{l+2}=O\left(n^{-l / 2}\right)$ и $\bar{z}_{k}=O(1)$. 
Далее мы определим модифицированное понятие кумулянтов случайной величины $W$. В [28] было показано, что в общем случае зависимых слагаемых разумно, вместо обычных кумулянтов $W$, рассматривать несколько иные характеристики. Это позволяет упростить представление и получить лучший остаточный член разложения. Кроме того, эти характеристики могут использоваться, когда традиционные приемы не работают. Пример этого дается в [28] и [30], и мы не будем повторять его здесь. Обозначим упомянутые характеристики $\bar{\gamma}_{k}$ и определим их следующим образом.

Так как $\mathbf{E} X_{i}=0$, определим первый модифицированный кумулянт как $\bar{\gamma}_{1}=0$. Перейдем к $\bar{\gamma}_{2}$. Если $X_{i}$ не зависит от $\widetilde{W}_{1 i}$ для каждого $i$, то, поскольку $\mathbf{E} X_{i}=0$, дисперсия $W$ будет равна $\sum_{i=1}^{n} \mathbf{E}\left\{X_{i} U_{1 i}\right\}$. Обозначим эту величину $\bar{\gamma}_{2}$ и будем рассматривать ее как модификацию дисперсии - второго кумулянта - в общем случае (т.е. и тогда, когда $X_{i}$ зависит от $\left.\widetilde{W}_{1 i}\right)$. Заметим, что $\bar{\gamma}_{2}$ зависит от выбора разложений (1.2.2). В случае локальной зависимости $\bar{\gamma}_{2}$ совпадает с $\mathbf{D} W$.

Таким образом, доказывая предельную теорему или устанавливая асимптотические разложения в ней, мы нормируем $W$ таким образом, чтобы единице была равна не дисперсия, а $\bar{\gamma}_{2}$.

Для того чтобы определить третий модифицированный кумулянт, мы опять должны рассмотреть случай локальной зависимости и написать выражение для третьего кумулянта $W$ в терминах смешанных моментов третьего порядка для случайных величин $X_{i}, U_{1 i}$ и $U_{2 i}$. Таким образом, мы получим определение $\bar{\gamma}_{3}$ в общем случае; и т.д. для всех $\bar{\gamma}_{k}$, $k=1, \ldots, d$, которые совпадают с соответствующими кумулянтами в случае локальной зависимости. Точная формула для $\bar{\gamma}_{k}$ будет приведена в п. 2.1 (формула (2.1.4)), а сейчас мы только отметим, что

$$
\left|\bar{\gamma}_{k}\right| \leqslant C(k) \bar{\mu}_{k}
$$

где константа $C(k)$ зависит только от $k$; см. п. 2.1.

В случае (1.2.11) отсюда следует, что $\bar{\gamma}_{2}=O(1), \bar{\gamma}_{l+2}=O\left(n^{-l / 2}\right)$, для $l \geqslant 0$.

1.3. Результат для гладких функций $h$. Определим (см., например, [5], [26]) знакопеременную меру

$$
Q_{\nu}(d x)=\sum_{(\nu)} p_{\mathbf{k} \nu} L_{\nu+2 s}(d x),
$$

где $L_{m}(d x)=(-1)^{m} \varphi^{(m)}(x) d x, \varphi(x)$ означает стандартную нормальную плотность,

$$
p_{\mathrm{k} \nu}=\prod_{m=1}^{\nu} \frac{1}{k_{m} !}\left(\frac{\bar{\gamma}_{m+2}}{(m+2) !}\right)^{k_{m}},
$$

$s=s(\mathbf{k})=k_{1}+\cdots+k_{\nu}$ и суммирование в $\sum_{(\nu)}$ ведется по всем векторам $\mathbf{k}=\left(k_{1}, \ldots, k_{\nu}\right)$ неотрицательных целых чисел таких, что 
$k_{1}+2 k_{2}+\cdots+\nu k_{\nu}=\nu$. Отличие от стандартной ситуации состоит в том, что в (1.3.2) вместо кумулянтов случайной величины $W$ мы используем характеристики $\bar{\gamma}_{k}$.

Пусть для фиксированного выше $d$

$$
\bar{\nu}_{l}=\sum_{i=1}^{n} \mu_{d+1, i}^{l /(d+1)} \quad \text { и } \quad \bar{\Pi}_{d}=\sum_{l=1}^{d} \bar{\nu}_{l} K^{1-l /(d+1)} .
$$

Эквивалентный способ определения $\bar{\Pi}_{d}-$ это положить

$$
\Pi_{d i}=\sum_{l=1}^{d} \mu_{d+1, i}^{l /(d+1)} K^{1-l /(d+1)} \quad \text { и } \quad \bar{\Pi}_{d}=\sum_{i=1}^{n} \Pi_{d i}
$$

(таким образом, черта опять означает суммирование характеристик отдельных слагаемых).

Чтобы определить порядок $\bar{\Pi}_{d}$, предположим опять, что (1.2.11) выполняется и $U_{i}$ являются суммами ограниченного числа $X$-ов. Тогда, как легко видеть, $\mu_{d+1, i}^{1 /(d+1)}=O\left(n^{-1 / 2}\right)$, и, следовательно,

$$
\bar{\nu}_{1}=O(\sqrt{n}), \quad \bar{\nu}_{2}=O(1) \quad \text { и } \quad \bar{\nu}_{l}=O\left(n^{-(l-2) / 2}\right) \quad \text { для } \quad l>2 .
$$

Таким образом,

$$
\begin{aligned}
\bar{\Pi}_{d}=O( & \sqrt{n} K^{d /(d+1)}+K^{(d-1) /(d+1)}+\frac{1}{\sqrt{n}} K^{(d-2) /(d+1)} \\
& \left.+\frac{1}{n} K^{(d-3) /(d+1)}+\cdots+\frac{1}{n^{(d-2) / 2}} K^{1 /(d+1)}\right) .
\end{aligned}
$$

Слагаемые в (1.3.4) представляют своего рода «взаимообмен» между степенями $n$ и $K$. (Последняя характеристика обычно зависит от $n$.) Легко проверить, что если $K=K(n)=o\left(n^{-1 /(2(d+1))}\right)$, то последний член является наихудшим. Далее мы увидим, как это влияет на точность разложения.

Пусть

$$
\widehat{h}_{p}=\max _{k=0, \ldots, p}\left\|h^{(k)}\right\|_{\infty}
$$

где $h^{(k)}-k$-я производная $h$, и далее везде допустим, что $W$ уже нормирована таким образом, что

$$
\bar{\gamma}_{2}=1 \text {. }
$$

Теорема 1. Существует некоторая константа $C(d)$ такая, что

$$
\mathbf{E} h(W)-\mathbf{\Phi}(h)=\sum_{\nu=1}^{d-2} \int h(x) Q_{\nu}(d x)+R_{d},
$$

где $\mathbf{\Phi}(h)=\int h(x) \Phi(d x) u$

$$
\left|R_{d}\right| \leqslant C(d) \widehat{h}_{d-1}\left(1+\bar{z}_{d}\right)^{d-2}\left[\bar{\mu}_{d+1}+\bar{\Pi}_{d}\right] .
$$


Чтобы пояснить приведенный выше результат, снова рассмотрим случай (1.2.11) и ограниченное число слагаемых $U$.

Для краткости обозначим последнее слагаемое главного члена разложения (1.3.6), т.е. $\int h(x) Q_{d-2}(d x)$, символом $\Gamma_{n}$. В рассматриваемом случае $\Gamma_{n}=O\left(n^{-(d-2) / 2}\right)$, и для больших $n$ это наименьшее слагаемое в главном члене разложения.

Теперь рассмотрим порядок остаточного члена $R_{d}$. Как мы уже видели, $z_{d}=O(1)$. Заметим, что в общей ситуации член $\left(1+\bar{z}_{d}\right)^{d-2}$ в (1.3.7) также ограничен и близок к $\left(1+\bar{\mu}_{2}\right)^{d-2}$ благодаря нормировке и так как $\bar{\mu}_{k}$ «мало» для $k \geqslant 3$.

Член $\bar{\mu}_{d+1}$ в (1.3.7) имеет порядок $O\left(n^{-(d-1) / 2}\right)$.

Рассмотрим последний член остатка, т.е. $\bar{\Pi}_{d}$. Мы видим, что если $K=K(n)=o\left(n^{-1 /(2(d+1))}\right)$, то $\bar{\Pi}_{d}=O\left(n^{-(d-2) / 2} K^{1 /(d+1)}\right)$, т.е. в любом случае остаток для больших $n$ меньше, чем $\Gamma_{n}$. Как легко вычислить, для $K=o\left(n^{-(d+1) / 2}\right)$, что является мягким требованием, $\bar{\Pi}_{d}=o\left(n^{-(d-1) / 2}\right)$, т.е. $\bar{\Pi}_{d}$ меньше, чем $\bar{\mu}_{d+1}$, для больших $n$, и весь остаточный член имеет порядок $\bar{\mu}_{d+1}=O\left(n^{-(d-1) / 2}\right)$.

Интересно сравнить этот результат с результатом из [28]. В случае (1.2.11) аналог $\bar{\Pi}_{d}$ в [28] имеет порядок $\sqrt{n} T$, где $T$ определено соотношением (1.2.4). «Плата» за переход к лучшей характеристике $K$ состоит в том, что мы возводим $K$ в степень меньшую единицы (хотя и умножаем $K$ на $\left.O\left(n^{-(d-2) / 2}\right)\right)$. Если, например, $K=K(n)$ убывает экспоненциально, то, так как $\bar{\mu}_{d+1}$ убывает как степенная функция, указанное обстоятельство не влияет на порядок остатка.

1.4. Случай, когда распределение $W$ гладкое. В этом случае условие гладкости $h$ может быть ослаблено. Рассмотрим слагаемое $X=X_{i}$, опустив на время индекс $i$. Обозначим $q_{s}(\cdot, \omega)$ условную плотность $\widetilde{W}_{s}$ относительно $\mathscr{F}_{s}$, полагая, что она существует для п.в. $\omega$ из вероятностного пространства. Мы опустим стандартные вопросы неединственности и регулярности. Если для некоторого $m, 1 \leqslant m \leqslant d-1$, плотность $q_{s}(x, \omega)$ является $m$ раз дифференцируемой по $x$, то положим

$$
\psi_{m}=\max _{s=0, \ldots, d} \operatorname{ess} \sup _{\omega}\left\{\max _{k=0, \ldots, m-1}\left\|q_{s}^{(k)}(\cdot, \omega)\right\|_{\infty}+\max _{k=0, \ldots, m}\left\|q_{s}^{(k)}(\cdot, \omega)\right\|_{1}\right\} .
$$

Заметим, что так как $q^{(0)}$ - плотность, то $\psi_{m} \geqslant 1$. Пусть $\psi_{0} \equiv 1$.

В основном утверждении, приведенном ниже, индекс $m$ в $\psi_{m}$ играет роль свободного параметра, и случай, когда плотность $q_{s}(\cdot, \omega)$ не существует, соответствует выбору $m=0$.

Восстанавливая индекс $i$, мы напишем $\psi_{m}=\psi_{m i}$ и положим

$$
\Psi_{m}=\max _{1 \leqslant i \leqslant n} \psi_{m i}
$$

Ниже мы будем считать, что величины $\Psi_{m},\left\|h^{(k)}\right\|_{\infty}$ и т.д. равны бесконечности не только, когда они бесконечны, но и когда они не су- 
ществуют. Это не вызовет недоразумения. Кроме того, условимся, что $\infty \cdot 0=0$.

Предположим теперь, что существует некоторое число $a=a(d)$ такое, что

$$
\omega(\varepsilon ; h) \leqslant a\|h\|_{\infty} \varepsilon,
$$

где $\omega(\varepsilon ; h)$ определена в (1.2.1). Это верно, например, если $h-$ индикатор интервала. Без потери общности предположим, что $a \geqslant 1$.

Положим $\widehat{\gamma}_{k}=\max \left\{\bar{\gamma}_{i} ; 2 \leqslant i \leqslant k\right\}$ и заметим, что в силу (1.3.5), $\widehat{\gamma}_{k} \geqslant 1$, и так как обычно $\bar{\gamma}_{i}$ мала для $i>2$, характеристика $\widehat{\gamma}_{k}$ обычно ограничена.

Теорема 2. Существует некоторая константа $C(d)$ такая, что разложение (1.3.6) выполняется с остаточным иленом $R_{d}$ таким, что для любого $m=0, \ldots, d-1$

$$
\left|R_{d}\right| \leqslant C(d) \widehat{h}_{d-1-m} \Psi_{m}\left(1+\bar{z}_{d}\right)^{d-2}\left\{\bar{\mu}_{d+1}+\left(\widehat{\gamma}_{d}^{d-2} a\right)^{1-1 / \widetilde{m}} \bar{\Pi}_{d}^{1 / \tilde{m}}\right\},
$$

где $\tilde{m}=\max \{1, m\}$. В случае $m=0,1$ условие (1.4.1) можно заменить условием (1.2.1).

Далее мы обсудим оценку (1.4.2). Если положить $m=0$, т.е. не требовать гладкости распределений $W_{s}$, то из оценки (1.4.2) мы получим теорему 1 . Если $m=1$, т.е. предположить, что все $W_{s}$ имеют ограниченные и интегрируемые условные плотности, то можно снизить требуемый порядок гладкости $h$ на единицу, т.е.

$$
\left|R_{d}\right| \leqslant C(d) \widehat{h}_{d-2} \Psi_{1}\left(1+\bar{z}_{d}\right)^{d-2}\left\{\bar{\mu}_{d+1}+\bar{\Pi}_{d}\right\}
$$

(cp. (1.3.7)).

Чем более гладко распределение $W_{s}$, тем ниже может быть порядок конечной производной $h$, но «плата» за это состоит в том, что мы возводим $\bar{\Pi}_{d}$ в степень меньшую единицы. В частности, если $\Psi_{d-1}$ конечно, то можно положить $m=d-1$ и рассмотреть любую ограниченную функцию $h$, для которой выполняется (1.4.1). Это приведет к оценке

$$
\begin{aligned}
\left|R_{d}\right| & \leqslant C(d)\|h\|_{\infty} \Psi_{d-1}\left(1+\bar{z}_{d}\right)^{d-2}\left\{\bar{\mu}_{d+1}+\left(\widehat{\gamma}_{d}^{d-2} a\right)^{(d-2) /(d-1)} \bar{\Pi}_{d}^{1 /(d-1)}\right\} \\
& \leqslant C(d)\|h\|_{\infty} \Psi_{d-1}\left(1+\bar{z}_{d}\right)^{d-2}\left(\widehat{\gamma}_{d}^{d-2} a\right)^{(d-2) /(d-1)}\left\{\bar{\mu}_{d+1}+\bar{\Pi}_{d}^{1 /(d-1)}\right\} .
\end{aligned}
$$

(Последнее неравенство верно, так как $\widehat{\gamma}_{d}^{d-2} \geqslant 1$ и $a \geqslant 1$.)

Если $\bar{z}_{d}, \widehat{\gamma}_{d}$ и $\Psi_{d-1}$ ограничены, то

$$
R_{d}=O\left(\bar{\mu}_{d+1}+\bar{\Pi}_{d}^{1 /(d-1)}\right) .
$$

В случае (1.2.11), если, например, $K=K(n)$ сходится к нулю экспоненциально, то $R_{d}=O\left(\bar{\mu}_{d+1}\right)$. 


\section{2. Доказательства}

2.1. Некоторые определения. Прежде всего мы напомним некоторые обозначения, введенные в [28] и [30]. Рассмотрим слагаемое $X=X_{i}$. На время (если это это не приведет к недоразумению) опустим индекс $i$ в $X_{i}$ и в соответствующих членах разложения $W_{k i}, U_{s i}$ и моментах $\mu_{k i}$ (см. п. 1.2).

Имеем $W_{k}=U_{1}+\cdots+U_{k}, W=W_{k}+\widetilde{W}_{k}$. Положим

$$
\mathbf{V}_{s}=\left(W_{s}, U_{s+1}, \ldots, U_{r}, U_{r+1}\right), \quad \mathbf{U}=\mathbf{V}_{0}=\left(U_{1}, \ldots, U_{r}, U_{r+1}\right) .
$$

Для $s+l \leqslant r+1, p \leqslant l$ определим

$$
\chi_{l}(s, p)=\sum_{|\mathbf{m}|=l}^{(p)} \frac{1}{\mathbf{m} !} \mathbf{E V}_{s}^{\mathbf{m}} \quad \text { и } \quad \vartheta_{l+1}(p)=\sum_{|\mathbf{m}|=l}^{(p)} \frac{1}{\mathbf{m} !} \mathbf{E}\left\{X \mathbf{U}^{\mathbf{m}}\right\},
$$

где $\sum_{|\mathbf{m}|=l}^{(p)}-$ сумма по всем $\mathbf{m}=\left(m_{1}, \ldots, m_{r+1}\right)$ таким, что $m_{i}=$ $1, \ldots, r+1$ для всех $i \leqslant p, m_{i}=0$ для $p<i \leqslant r+1$ и $|\mathbf{m}|=m_{1}+$ $\cdots+m_{r+1}=l$; кроме того, $\mathbf{U}^{\mathrm{m}}=\prod_{i=1}^{r+1} U_{i}^{m_{i}}, \mathbf{V}_{s}^{\mathrm{m}}=W_{s}^{m_{1}} U_{s+1}^{m_{2}} \cdots U_{r+1}^{m_{r-s+2}}$ и $\mathbf{m} !=m_{1} ! \cdots m_{r+1} !$. Нетрудно видеть, что

$$
\begin{aligned}
\left|\chi_{l}(s, p)\right| & \leqslant \sum_{|\mathbf{m}|=l}^{(p)} \frac{1}{\mathbf{m} !} \mathbf{E}\left|\mathbf{V}_{s}^{\mathbf{m}}\right| \leqslant \frac{1}{l !} \mathbf{E}\left\{\left(\left|W_{s}\right|+\left|U_{s+1}\right|+\cdots+\left|U_{s+l-1}\right|\right)^{l}\right\} \\
& \leqslant \frac{1}{l !} \mathbf{E}\left\{\left(\left|U_{1}\right|+\cdots+\left|U_{s+l-1}\right|\right)^{l}\right\} \leqslant \frac{1}{l !} \mathbf{E}\left\{\left(\left|U_{1}\right|+\cdots+\left|U_{r}\right|\right)^{l}\right\} \\
& \leqslant \mathbf{E}\left\{\left(|X|+\left|U_{1}\right|+\cdots+\left|U_{r+1}\right|\right)^{l}\right\}=\mu_{l} .
\end{aligned}
$$

Аналогично

$$
\left|\vartheta_{l}(s)\right| \leqslant \mu_{l}
$$

Пусть $\Upsilon_{00}=1, \Upsilon_{l 0}=0$ для $l \geqslant 1$ и для $m \geqslant 1$

$$
\begin{aligned}
& \Upsilon_{l m}=\Upsilon_{l m}(s) \\
&=\sum_{l_{1}+\cdots+l_{m}=l} \sum_{p_{1}=1}^{l_{1}} \cdots \sum_{p_{m}=1}^{l_{m}} \chi_{l_{1}}\left(s, p_{1}\right) \chi_{l_{2}}\left(s+p_{1}, p_{2}\right) \\
& \cdots \chi_{l_{m}}\left(s+p_{1}+\cdots+p_{m-1}, p_{m}\right) .
\end{aligned}
$$

Для $l \leqslant r$ попожим

$$
\begin{gathered}
c(s, l)=\sum_{m=0}^{[l / 2]}(-1)^{m} \Upsilon_{l m}(s), \quad \widetilde{c}(s, l)=\sum_{m=0}^{[l / 2]}\left|\Upsilon_{l m}(s)\right| \\
(c(s, 0)=1, \quad c(s, 1)=1, s \geqslant 0) .
\end{gathered}
$$


Вспоминая, что приведенные выше величины зависят от индекса $i$, запишем

$$
\frac{\gamma_{m+1, i}}{m !}=\sum_{t+l=m, l \geqslant 1} \sum_{s=1}^{l} \vartheta_{l+1, i}(s) c_{i}(s+1, t), \quad \bar{\gamma}_{m+1}=\sum_{i=1}^{n} \gamma_{m+1, i},
$$

$m=1, \ldots, r$.

Последняя формула дает формальное определение величин $\bar{\gamma}_{m}$, введенных в п. 1.2.4. Заметим также, что, как нетрудно видеть,

$$
\begin{aligned}
& \mu_{l} \mu_{k} \leqslant \mu_{l+k}, \quad \text { и, следовательно, } \\
& \Upsilon_{l m}, c(s, l), \widetilde{c}(s, l) \leqslant C(l) \mu_{l} \quad \text { для всех } l \leqslant \mathrm{r}, \\
& \gamma_{m+1} \leqslant C(m) \mu_{m+1}, \quad \bar{\gamma}_{m+1} \leqslant C(m) \bar{\mu}_{m+1}
\end{aligned}
$$

для констант $C(l)$, зависящих только от $l$.

2.2. Основное представление. Допустим, на время, что $f-$ произвольная достаточно гладкая функция. Положим

$$
\begin{aligned}
\hat{f}_{l} & =\max _{k \leqslant l}\left\|f^{(k)}\right\|_{\infty}, \\
\Gamma_{l}(f) & =\max _{1 \leqslant i \leqslant n} \max _{s \leqslant r+1} \sup _{x} \operatorname{ess~sup}_{\omega}\left|\mathbf{E}\left\{f^{(l)}\left(\widetilde{W}_{s i}+x\right) \mid \mathscr{F}_{s i}\right\}(\omega)\right|,
\end{aligned}
$$

при условии, что приведенные выше величины корректно определены. Очевидно, что $\Gamma_{l}(f) \leqslant \hat{f}_{l}$.

Пусть для целого $r \leqslant d$

$$
\bar{\Pi}_{r}=\sum_{l=1}^{r} \bar{\nu}_{l} K^{1-l /(d+1)}
$$

где $\bar{\nu}_{l}$ определено в (1.3.3). Заметим, что (2.2.2) совпадает с определением $\bar{\Pi}_{d}$ из (1.3.3).

Доказательства теорем, приведенных выше, опираются на следующую формулу.

Предложение 1. Если $\mathbf{E} X_{i}=0 u \mathbf{E}\left|X_{i}\right|^{d+1}<\infty$ для всех $i$, mо $\partial л я$ любого иелого $r \leqslant d-1$ и достаточно гладкой $f$

$$
\mathbf{E}\{W f(W)\}=\sum_{m=1}^{r} \frac{\bar{\gamma}_{m+1}}{m !} \mathbf{E} f^{(m)}(W)+B_{r}(f)
$$

гдe

$$
\left|B_{r}(f)\right| \leqslant C(r)\left[\bar{\mu}_{r+2} \Gamma_{r+1}(f)+\hat{f}_{r} \bar{\Pi}_{r+1}\right] .
$$

Предложение 1 используется для получения разложений Эджворта следуюшим образом. Напомним, что $\mathbf{E} X_{i}=0, \bar{\gamma}_{2}=1$. Тогда из (2.2.3) следует, что

$$
\mathbf{E}\left\{f^{\prime}(W)-W f(W)\right\}=-\sum_{m=2}^{r} \frac{\bar{\gamma}_{m+1}}{m !} \mathbf{E} f^{(m)}(W)-B_{r}(f) .
$$


Пусть $f$ - функция Стейна, являющаяся решением дифференциального уравнения $f^{\prime}(w)-w f(w)=h(w)-\boldsymbol{\Phi}(h)$ (см., например, [34], [35]), т.е.

$$
f(x)=\frac{1}{\varphi(x)} \int_{-\infty}^{x}[h(t)-\boldsymbol{\Phi}(h)] \varphi(t) d t .
$$

Тогда

$$
\mathbf{E} h(W)-\boldsymbol{\Phi}(h)=-\sum_{m=2}^{r} \frac{\bar{\gamma}_{m+1}}{m !} \mathbf{E} f^{(m)}(W)-B_{r}(f)
$$

Следующий (достаточно длинный) этап состоит в выполнении индукции относительно порядка разложений, причем индукционное предположение применяется к членам $\mathbf{E} f^{(m)}(W)$ в (2.2.7). Это сделано в п. 2.3.2.

Предложение 1 очень похоже на предложение 3.12 из [30], но немного отличается от последнего определением $\bar{\Pi}_{r}$. Упомянутые предложения не сравнимы: для каждого из них существует ситуация, когда другое оказывается сильнее. Однако доказательство соотношений $(2.2 .3),(2.2 .4)$ практически такое же, как и доказательство в [30]. Поэтому здесь мы только перечислим то, что надо изменить в доказательстве из [30]. Фактически, надо сделать только одну вещь: во всем доказательстве в [30] заменить члены типа $\mu_{r+2}^{l /(r+2)} K^{1-l /(r+2)}$ (где $r$ переменный параметр) на $\mu_{d+1}^{l /(d+1)} K^{1-l /(d+1)}$ (где $d$ - фиксированный параметр). Это приведет к следующему.

1. Определение (5.10) в [30] должно быть заменено на $\Pi_{k}=\Pi_{k i}=$ $\sum_{l=1}^{k} \mu_{d+1, i}^{l /(d+1)} K^{1-l /(d+1)}$.

2. Неравенство (5.11) из [30], которое следует из этого определения и где применено неравенство Давыдова из [10], должно быть заменено на

$$
\begin{aligned}
\left|T_{r}\right| & \leqslant 8 \mu_{d+1}^{1 /(d+1)} \hat{f}_{0} K^{1-1 /(d+1)}+8 \sum_{l=1}^{r} \sum_{s=1}^{l} \sum_{|\mathbf{m}|=l}{ }^{(s)} \frac{1}{\mathbf{m} !} \mu_{d+1}^{(l+1) /(d+1)} \hat{f}_{l} K^{1-(l+1) /(d+1)} \\
& \leqslant C(r) \hat{f}_{r} \Pi_{r+1} .
\end{aligned}
$$

3. Последние четыре неравенства на с. 81 в [30] должны выглядеть следующим образом:

$$
\begin{aligned}
& \leqslant C(r)\left\{\mu_{r+2} \Gamma_{r+1}(f)+\hat{f}_{r} \Pi_{r+1}+\hat{f}_{r} \sum_{l=1}^{r} \mu_{r+2}^{(l+1) /(r+2)} \sum_{s=1}^{r-l} \mu_{r+2}^{s /(r+2)} K^{1-s /(r+2)}\right\} \\
& \leqslant C(r)\left\{\mu_{r+2} \Gamma_{r+1}(f)+\hat{f}_{r} \Pi_{r+1}+\hat{f}_{r} \sum_{l=1}^{r} \sum_{s=1}^{r-l} \mu_{d+1}^{(l+s+1) /(d+1)} K^{1-(l+s+1) /(d+1)}\right\} \\
& \leqslant C(r)\left\{\mu_{r+2} \Gamma_{r+1}(f)+\hat{f}_{r} \Pi_{r+1}+\hat{f_{r}} \sum_{l=1}^{r} \sum_{p=l+2}^{r+1} \mu_{d+1}^{p /(d+1)} K^{1-p /(d+1)}\right\} \\
& \leqslant C(r)\left\{\mu_{r+2} \Gamma_{r+1}(f)+\hat{f}_{r} \Pi_{r+1}\right\} .
\end{aligned}
$$


4. В первой формуле на с. $82 K(r)$ должно быть заменено на $\Pi_{r+1}$.

5. Первое неравенство на с. 83 должно выглядеть так:

$$
\begin{aligned}
\left|T_{k s}(f)\right| & \leqslant 8 \sum_{l=1}^{k} \sum_{p=1}^{l} \sum_{|\mathbf{m}|=l}(p) \frac{1}{\mathbf{m} !}\left\|\mathbf{V}_{s}^{\mathbf{m}}\right\|_{(r+2) / l} \hat{f}_{l} K^{1-l /(d+1)} \\
& \leqslant 8 \hat{f}_{k} \sum_{l=1}^{k} \sum_{p=1}^{l} \sum_{|\mathbf{m}|=l}{ }^{(p)} \frac{1}{\mathbf{m} !} \mu_{d+1}^{l /(d+1)} K^{1-l /(d+1)} \leqslant C(k) \hat{f}_{k} \Pi_{k} .
\end{aligned}
$$

6. Последняя цепочка неравенств на с. 84 должна быть изменена следующим образом:

$$
\begin{aligned}
\left|M_{s k}(f)\right| & \leqslant C(k)\left(\Gamma_{k+1}(f) \sum_{l=0}^{k} \mu_{l} \mu_{k+1-l}+\hat{f}_{k} \sum_{l=0}^{k} \mu_{l} \sum_{s=1}^{k-l} \mu_{d+1}^{s /(d+1)} K^{1-s /(d+1)}\right) \\
& \leqslant C(k)\left(\Gamma_{k+1}(f) \mu_{k+1}+\hat{f}_{k} \sum_{l=0}^{k} \sum_{s=1}^{k-l} \mu_{d+1}^{(s+l) /(d+1)} K^{1-s /(d+1)}\right) \\
& \leqslant C(k)\left(\Gamma_{k+1}(f) \mu_{k+1}+\hat{f}_{k} \sum_{l=0}^{k} \sum_{s=1}^{k-l} \mu_{d+1}^{(s+l) /(d+1)} K^{1-(s+l) /(d+1)}\right) \\
& \leqslant C(k)\left(\Gamma_{k+1}(f) \mu_{k+1}+\hat{f}_{k} \sum_{l=0}^{k} \sum_{p=1+l}^{k} \mu_{d+1}^{p /(d+1)} K^{1-p /(d+1)}\right) \\
& \leqslant C(k)\left(\Gamma_{k+1}(f) \mu_{k+1}+\hat{f}_{k} \Pi_{k}\right) .
\end{aligned}
$$

2.3. Доказательство теоремы 2. Прежде всего мы докажем следуюшую теорему.

Теорема 3. Существует константа $C(d)$ такая, что разложение (1.3.6) справедливо с

$$
\left|R_{d}\right| \leqslant C(d)\left(1+\bar{z}_{d}\right)^{d-2}\left\{\widehat{h}_{d-1-m} \Psi_{m} \bar{\mu}_{d+1}+\widehat{h}_{d-2} \bar{\Pi}_{d}\right\}
$$

для всех $m=0, \ldots, d-1$.

Доказательство этой теоремы повторяет доказательство теоремы 1 из [28], но несколько сложнее. Для полноты картины мы вкратце повторим некоторые моменты из [28].

Прежде всего, нам необходимы следующие леммы.

\subsection{1. Леммы.}

Лемма 1 ([28]). Для $k, l \geqslant 2$

$$
\bar{\mu}_{k} \bar{\mu}_{l} \leqslant \bar{\mu}_{k+l-2} \bar{\mu}_{2}
$$

Везде далее $f$ обозначает функцию Стейна из (2.2.6).

Лемма 2. Для любого $k \geqslant 1$ выполнено неравенство $\left\|f^{(k)}\right\|_{\infty} \leqslant$ $C(k)\left\|h^{(k-1)}\right\|_{\infty}$, где константа $C(k)$ зависит только от $k$. 
Доказательство можно найти в [1]; для $k=1,2-$ в [35]. Далее, заметим, что $f$ можно представить как (см. [1])

$$
\begin{aligned}
f(x)= & \left(-I(x \geqslant 0) \int_{0}^{\infty}+I(x<0) \int_{-\infty}^{0}\right) \\
& \times e^{-x z-z^{2} / 2}[h(x+z)-\boldsymbol{\Phi}(h)] d z .
\end{aligned}
$$

Дифференцируя (2.3.3), для $k \geqslant 1$ получим, что

$$
\begin{aligned}
f^{(k)}(x)= & (-1)^{k-1} \boldsymbol{\Phi}(h) G(x ; 1 ; k, 0) \\
& +\sum_{m=0}^{k}(-1)^{k-m}\left(\begin{array}{c}
k \\
m
\end{array}\right) G(x ; h ; k-m, m),
\end{aligned}
$$

где

$G(x ; h ; l, m)=\left(-I(x \geqslant 0) \int_{0}^{\infty}+I(x<0) \int_{-\infty}^{0}\right) z^{l} e^{-x z-z^{2} / 2} h^{(m)}(x+z) d z$.

Положим $\boldsymbol{\Phi}_{k}(h)=\int_{-\infty}^{\infty} z^{k} h(z) \varphi(z) d z\left(\right.$ так что $\left.\boldsymbol{\Phi}(h)=\boldsymbol{\Phi}_{0}(h)\right)$ и для случайной величины $Y$ определим

$$
\begin{aligned}
\Lambda_{r}(h ; Y)= & \max _{1 \leqslant t \leqslant r^{3}, l \leqslant r^{3}, \nu \leqslant r}\left|\mathbf{E}\left\{Y^{t} G(Y ; h ; l+t-1, \nu)\right\}\right| \\
& +\max _{l \leqslant r^{3}, \nu \leqslant r}|\mathbf{E} G(Y ; h ; l, \nu)|+\left|\mathbf{E} h^{(r)}(Y)\right|+\max _{l \leqslant r^{3}}\left|\mathbf{\Phi}_{l}(h)\right| .
\end{aligned}
$$

Сложное выражение в (2.3.5) и, в частности, член $r^{3}$ необходимы для индукции в доказательствах двух следующих лемм из [28].

Лемма 3 ([28]). Для любого челого $r \geqslant 2$, достаточно гладкой функции $h$ и случайной величинь $Y$

$$
\left|\mathbf{E} f^{(r+1)}(Y)\right| \leqslant C(r) \Lambda_{r}(h ; Y),
$$

$u \partial \Omega s p=2, \ldots, r$

$$
\Lambda_{r+1-p}\left(f^{(p)} ; Y\right) \leqslant C(r) \Lambda_{r}(h ; Y) .
$$

Лемма 4 ([28]). Пусть $Y-$ случайная величина с плотностью $p(x)$ такая, что для некоторого $m, 1 \leqslant m \leqslant r$,

$$
p_{m}:=\max _{k \leqslant m-1}\left\|p^{(k)}\right\|_{\infty}+\max _{k \leqslant m}\left\|p^{(k)}\right\|_{1}<\infty .
$$

Тогда

$$
\Lambda_{r}(h ; Y) \leqslant C(r) p_{m} \max _{k=0, \ldots, r-m}\left\|h^{(k)}\right\|_{\infty} .
$$

Для $m=0$ оценка (2.3.8) всегда верна с $p_{0}=1$.

Теперь мы можем перейти непосредственно к доказательству теоремы 3 . 
2.3.2. Доказательство теоремы 3. Пусть $\Lambda_{r}(h ; Y ; i, s, \omega)$ - это величина $\Lambda_{r}(h ; Y)$ в $(2.3 .5)$, где математическое ожидание $\mathbf{E}$ заменено на $\mathbf{E}\left\{\cdot \mid \mathscr{F}_{s i}\right\}$. Тогда, учитывая (2.3.6),

$\Gamma_{r+1}(f) \leqslant C(r) \max _{1 \leqslant i \leqslant n} \max _{s \leqslant r+1} \sup _{x} \operatorname{ess} \sup _{\omega} \Lambda_{r}\left(h ; \widetilde{W}_{s i}+x ; i, s, \omega\right)=: C(r) \Lambda_{r}(h)$.

Поэтому

$$
\begin{aligned}
\left|B_{r}(f)\right| & \leqslant C(r)\left[\bar{\mu}_{r+2} \Gamma_{r+1}(f)+\hat{f}_{r} \bar{\Pi}_{r+1}\right] \\
& \leqslant C(r)\left[\bar{\mu}_{r+2} \Lambda_{r}(h)+\widehat{h}_{r-1} \bar{\Pi}_{r+1}\right]
\end{aligned}
$$

в силу леммы 2 .

Прежде чем доказывать теорему 3 , мы докажем следующее более сложное и более сильное утверждение: для $1 \leqslant t \leqslant d-1$

$$
\mathbf{E} h(W)-\mathbf{\Phi}(h)=\sum_{\nu=1}^{t-1} \int h(x) Q_{\nu}(d x)+K_{t}(h)
$$

или

$$
\mathbf{E} h(W)=\sum_{\nu=0}^{t-1} \int h(x) Q_{\nu}(d x)+K_{t}(h)
$$

где $Q_{0}(d x)=\Phi(d x)$ и

$$
\left|K_{t}(h)\right| \leqslant C(t)\left(1+\bar{z}_{t+1}\right)^{t-1}\left[\bar{\mu}_{t+2} \Lambda_{t}(h)+\widehat{h}_{t-1} \bar{\Pi}_{t+1}\right] .
$$

Заметим, что случай $t=1$ уже был доказан - см. (2.2.7) и (2.3.10) (для $r=1$, используя также соотношение $h(W)-\mathbf{\Phi}(h)=f^{\prime}(W)-W f(W)$ и лемму 2). Следовательно, нам осталось доказать по индукции случай $t=r$, полагая, что соотношения (2.3.12), (2.3.13) справедливы для $1 \leqslant$ $t \leqslant r-1$. Учитывая (2.2.7), затем по гипотезе индукции, примененной к $f^{(p)}$ для $t-1=r-p$, и окончательно по (1.3.1), получаем

$$
\begin{aligned}
\mathbf{E} h(W)-\boldsymbol{\Phi}(h)= & -\sum_{p=2}^{r} \frac{\bar{\gamma}_{p+1}}{p !}\left(\sum_{\nu=0}^{r-p} \int f^{(p)}(x) Q_{\nu}(d x)+K_{r-p+1}\left(f^{(p)}\right)\right) \\
& -B_{r}(f)=-\sum_{p=2}^{r} \frac{\bar{\gamma}_{p+1}}{p !} \sum_{\nu=0}^{r-p} \sum_{(\nu)} p_{\mathbf{k} \nu} \int f^{(p)}(x) L_{\nu+2 s}(d x) \\
& -\sum_{p=2}^{r} \frac{\bar{\gamma}_{p+1}}{p !} K_{r-p+1}\left(f^{(p)}\right)-B_{r}(f) .
\end{aligned}
$$

Нетрудно видеть, что из (2.3.7) следует, что

$$
\Lambda_{r+1-p}\left(f^{(p)}\right) \leqslant C(r) \Lambda_{r}(h) .
$$


Используя (2.1.5), леммы 1 и 2, неравенство (2.3.15) и гипотезу индукции для $K_{r-p+1}\left(f^{(p)}\right)$, и снова допуская, что $C(r)$ различна для разных соотношений и зависит только от $r$, мы получим

$$
\begin{aligned}
& \sum_{p=2}^{r}\left|\bar{\gamma}_{p+1} K_{r-p+1}\left(f^{(p)}\right)\right| \\
& \quad \leqslant C(r) \sum_{p=2}^{r} \bar{\mu}_{p+1}\left(1+\bar{z}_{r-p+2}\right)^{r-p}\left[\bar{\mu}_{r-p+3} \Lambda_{r+1-p}\left(f^{(p)}\right)+\hat{f}_{r} \bar{\Pi}_{r-p+2}\right] \\
& \quad \leqslant C(r)\left(1+\bar{z}_{r}\right)^{r-2}\left[\bar{\mu}_{2} \bar{\mu}_{r+2} \Lambda_{r}(h)+\widehat{h}_{r-1} \sum_{p=2}^{r} \bar{\mu}_{p+1} \bar{\Pi}_{r-p+2}\right]
\end{aligned}
$$

Для последней суммы имеем

$$
\begin{aligned}
\sum_{p=2}^{r} \bar{\mu}_{p+1} \bar{\Pi}_{r-p+2} & =\sum_{p=2}^{r} \bar{\mu}_{p+1} \sum_{l=1}^{r-p+2} \bar{\nu}_{l} K^{1-l /(d+1)} \\
& =\bar{\nu}_{1} K^{1-1 /(d+1)}\left(\sum_{p=2}^{r} \bar{\mu}_{p+1}\right)+\sum_{p=2}^{r} \bar{\mu}_{p+1} \sum_{l=2}^{r-p+2} \bar{\nu}_{l} K^{1-l /(d+1)} \\
& =\bar{\nu}_{1} K^{1-1 /(d+1)} \sum_{p=2}^{r} \bar{\mu}_{p+1}+\sum_{l=2}^{r} \bar{\nu}_{l} K^{1-l /(d+1)} \sum_{p=2}^{r-l+2} \bar{\mu}_{p+1} \\
& \leqslant \bar{\nu}_{1} K^{1-1 /(d+1)} \bar{z}_{r+1}+\sum_{l=2}^{r} \bar{\nu}_{l} K^{1-l /(d+1)} \bar{z}_{r-l+3} \leqslant \bar{z}_{r+1} \bar{\Pi}_{r}
\end{aligned}
$$

и, следовательно,

$$
\sum_{p=2}^{r}\left|\bar{\gamma}_{p+1} K_{r-p+1}\left(f^{(p)}\right)\right| \leqslant C(r)\left(1+\bar{z}_{r+1}\right)^{r-1}\left(\bar{\mu}_{r+2} \Lambda_{r}(h)+\widehat{h}_{r-1} \bar{\Pi}_{r}\right) .
$$

Учитывая (2.3.10), получаем следующую оценку:

$$
\begin{aligned}
\left|K_{r}(h)\right| \leqslant & C(r)\left[\bar{\mu}_{r+2} \Lambda_{r}(h)+\widehat{h}_{r-1} \bar{\Pi}_{r+1}\right] \\
& +C(r)\left(1+\bar{z}_{r+1}\right)^{r-1}\left(\bar{\mu}_{r+2} \Lambda_{r}(h)+\widehat{h}_{r-1} \bar{\Pi}_{r}\right) \\
\leqslant & C(r)\left(1+\bar{z}_{r+1}\right)^{r-1}\left[\bar{\mu}_{r+2} \Lambda_{r}(h)+\widehat{h}_{r-1} \bar{\Pi}_{r+1}\right],
\end{aligned}
$$

которая завершает индукцию в доказательстве (2.3.13).

Далее мы положим в (2.3.11) и (2.3.13) $t=d-1$ и применим лемму 4 для оценки $\Lambda_{r}(h)$ и таким образом получим (2.3.1). (Роль плотности $p(x)$ в $(2.3 .8)$ играет $q_{s}(x, \omega)$.)

Остальное доказательство точно повторяет соответствующую часть доказательства теоремы 2 в [28].

2.4. Сглаживание и доказательство теоремы 2. Далее мы выполним сглаживание. Суть состоит в том, что, как мы увидим, 
ошибка, возникаюшая при переходе к сглаженным функциям $h$, затрагивает только второй член в (2.3.1), т.е. наименьший член в остатке. Пусть $\mathbf{P}(d x)$ - распределение $W$ и знакопеременная мера

$$
Q(d x)=\sum_{\nu=0}^{d-2} Q_{\nu}(d x)=\Phi(d x)+\sum_{\nu=1}^{d-2} Q_{\nu}(d x) .
$$

Пусть для любой функции $h$ «сдвинутая» функция ${ }_{y} h(x)=h(x+y)$. Рассмотрим функцию $h$, функции $h_{\varepsilon+}, h_{\varepsilon-}$ (см. п. 1.2.1) и ${ }_{y} h_{\varepsilon+},{ }_{y} h_{\varepsilon-}$. Заметим, что порядок двух операций в ${ }_{y} h_{\varepsilon}$ и ${ }_{y} h_{\varepsilon}$ - не существен и

$$
\left\|h_{\varepsilon+}^{(k)}\right\|_{\infty} \leqslant\left\|h^{(k)}\right\|_{\infty}, \quad\left\|h_{\varepsilon-}^{(k)}\right\|_{\infty} \leqslant\left\|h^{(k)}\right\|_{\infty} .
$$

Положим

$$
\begin{aligned}
\delta_{\varepsilon \pm} & =\sup _{y}\left|\int_{y} h_{\varepsilon \pm}(x)\left[(\mathbf{P}-Q) * \Phi_{\varepsilon}\right](d x)\right| \\
\tau_{\varepsilon} & =\sup _{y} \int\left[{ }_{y} h_{\varepsilon+}(x)-{ }_{y} h_{\varepsilon-}(x)\right]|Q|(d x),
\end{aligned}
$$

где $|Q|(d x)$ - вариация меры $Q(d x)$ и $\Phi_{\varepsilon}$ - нормальная мера с нулевым средним и дисперсией $\varepsilon^{2}$. По лемме 11.4 из [5, с. 95] для некоторой константы $C$ справедливо неравенство

$$
\left|\int h d(\mathbf{P}-Q)\right| \leqslant C\left[\max \left\{\delta_{\varepsilon+}, \delta_{\varepsilon-}\right\}+\tau_{2 \varepsilon}\right] .
$$

Заметим, что

$$
\int_{y} h_{\varepsilon \pm}(x)\left[(\mathbf{P}-Q) * \Phi_{\varepsilon}\right](d x)=\int_{y} \widetilde{h}_{\varepsilon \pm}(x)(\mathbf{P}-Q)(d x),
$$

где сглаженная функция

$$
{ }_{y} \widetilde{h}_{\varepsilon \pm}(x)=\int_{y} h_{\varepsilon \pm}(x+\varepsilon z) \Phi(d z) .
$$

Как легко проверить, исходя из (1.3.1), (1.3.2), (1.4.1) и (2.4.1),

$$
\tau_{\varepsilon} \leqslant C(d) \widehat{\gamma}_{d}^{d-2} \omega(h ; \varepsilon) \leqslant C(d)\|h\|_{\infty} \widehat{\gamma}_{d}^{d-2} a \varepsilon .
$$

Далее мы применим теорему $3 \mathrm{\kappa} \int_{y} \widetilde{h}_{\varepsilon \pm}(x)(\mathbf{P}-Q)(d x)$. Заметим, что, учитывая $(2.4 .2)$ и $(2.4 .5)$,

$$
\left\|_{y} \tilde{h}_{\varepsilon+}^{(k)}\right\|_{\infty} \leqslant C(k) \varepsilon^{-s}\left\|_{y} h_{\varepsilon+}^{(k-s)}\right\|_{\infty} \leqslant C(k) \varepsilon^{-s}\left\|h^{(k-s)}\right\|_{\infty}
$$

для $s \leqslant k$. Далее, по теореме 3 и формулам (2.4.4) и (2.4.7)

$$
\delta_{\varepsilon \pm} \leqslant C(d)\left(1+\bar{z}_{d}\right)^{d-2}\left\{\widehat{h}_{d-1-m} \Psi_{m} \bar{\mu}_{d+1}+\varepsilon^{-(\tilde{m}-1)} \widehat{h}_{d-1-m} \bar{\Pi}_{d}\right\}
$$

где $\widetilde{m}=\max \{m, 1\}$. Появление $\widetilde{m}$ связано с тем, что порядок производной во втором члене (2.3.1) меньше, чем в первом члене. Это позволяет использовать меньшую степень $\varepsilon$. 
Из (2.4.8), (2.4.6), (2.4.4) и (2.4.3) следует, что

$$
\begin{aligned}
& \left|\int h d(\mathbf{P}-Q)\right| \\
& \quad \leqslant C(d)\left\{\left(1+\bar{z}_{d}\right)^{d-2} \widehat{h}_{d-1-m} \Psi_{m}\left[\bar{\mu}_{d+1}+\varepsilon^{-(\tilde{m}-1)} \bar{\Pi}_{d}\right]+\widehat{\gamma}_{d}^{d-2}\|h\|_{\infty} a \varepsilon\right\} \\
& \quad \leqslant C(d)\left\{\left(1+\bar{z}_{d}\right)^{d-2} \widehat{h}_{d-1-m} \Psi_{m}\left[\bar{\mu}_{d+1}+\varepsilon^{-(\tilde{m}-1)} \bar{\Pi}_{d}+\widehat{\gamma}_{d}^{d-2} a \varepsilon\right]\right\},
\end{aligned}
$$

так как $\Psi_{m} \geqslant 1$. Остается выбрать $\varepsilon$.

\section{СПИСОК ЛИТЕРАТУРЫ}

1. Barbour A.D. Asymptotic expansions based on smooth functions in the central limit theorem. - Probab. Theory Related Fields, 1986, v. 72, № 2, p. 289-303.

2. Barbour A.D., Karoński M., Ruciński A. A central limit theorem for decomposable random variables with applications to random graphs. - J. Combin. Theory, Ser. B, 1989 , v. 47, № 2 , p. 125-145.

3. Bening V.E. On the rate of convergence and asymptotic expansions for $U$-statistics under alternatives. - J. Math. Sci. (New York), 2000, v. 99, № 4, p. 1403-1407.

4. Bentkus V., Götze F., van Zwet W. R. An Edgeworth expansion for symmetric statistics. - Ann. Statist., 1997, v. 25, № 2, p. 851-896.

5. Bhattacharya R., Ranga Rao R. Normal Approximation and Asymptotic Expansions. Melbourne: Krieger, 1986, $291 \mathrm{p}$.

6. Bickel P. J., Götze F., van Zwet W.R. The Edgeworth expansion for $U$-statistics of degree two. - Ann. Statist., 1986, v. 14, № 4, p. 1463-1484.

7. Bloznelis M., Götze F. One-term Edgeworth expansion for finite population $U$-statistics of degree two. - Acta Appl. Math., 1999, v. 58, № 1-3, p. 75-90.

8. Bradley R. C. Basic properties of strong mixing conditions. - Dependence in Probability and Statistics: A Survey of Recent Results (Oberwolfach, 1985). Boston: Birkhäuser, 1985, p. 165-192. (Progr. Probab. Statist., v. 11.)

9. Bradley R. Introduction to Strong Mixing Conditions. V. 1. Technical Report. Bloomington: Department of Mathematics, Custom Publishing of IU, Indiana University, 2002.

10. Давыдов Ю. А. Принцип инвариантности для стационарных процессов. - Теория вероятн. и ее примен., 1970, т. 15, в. 3, с. 498-509.

11. Götze F., Hipp C. Asymptotic expansions for sums of weakly dependent random vectors. - Z. Wahrscheinlichkeitstheor. Verw. Geb., 1983, v. 64, № 2, p. 211-239.

12. Heinrich $L$. Some remarks on asymptotic expansions in the central limit theorem for m-dependent random variables. - Math. Nachr., 1985, v. 122, p. 151-155.

13. Heinrich L. Asymptotic expansions in the central limit theorem for a special class of $m$-dependent random fields. I. - Math. Nachr., 1987, v. 134, p. 83-106.

14. Heinrich L. Asymptotic expansions in the central limit theorem for a special class of $m$-dependent random fields. II. Lattice case. - Math. Nachr., 1990, v. 145, p. 309327.

15. Hipp C. Asymptotic expansions in the central limit theorem for compound and Markov processes. - Z. Wahrscheinlichkeitstheor. Verw. Geb., 1985, v. 69, № 3, p. 361-385.

16. Ибрагимов И. А. Некоторые предельные теоремы для стационарных в узком смысле вероятностных процессов. - Докл. АН СССР, 1959, т. 125, № 4, с. 711-714.

17. Iosifescu M., Theodorescu R. Random Processes and Learning. Berlin: SpringerVerlag, 1969, 304 p. (Grundlehren Math. Wiss., v. 150.) 
18. Jensen J. G. Asymptotic expansions for strongly mixing Harris recurrent Markov chains. - Scand. J. Statist., 1989, v. 16, № 1, p. 47-63.

19. Jensen J.G. A note on asymptotic expansions for sums over a weakly dependent random field with application to the Poisson and Strauss processes. - Ann. Inst. Statist. Math., 1993, v. 45, № 2, p. 353-360.

20. Королюк В. С., Боровских Ю. В. Разложения для $U$-статистик и функционалов Мизеса. - Укр. матем. журн., 1985, т. 37, № 4, с. 450-456.

21. Lahiri S. N. Refinements in asymptotic expansions for sums of weakly dependent random vectors. - Ann. Probab., 1993, v. 21, № 2, p. 791-799.

22. Loh W.-L. An Edgeworth expansion for $U$-statistics with weakly dependent observations. - Statist. Sinica, 1996, v. 6, № 1, p. 171-186.

23. Maesono $Y$. Asymptotic mean square errors of variance estimators for $U$-statistics and their Edgeworth expansions. - J. Japan Statist. Soc., 1998, v. 28, № 1, p. 1-19.

24. Малиновский В.K. О предельных теоремах для харрисовских цепей Маркова, I. - Теория вероятн. и ее примен., 1986, т. 31, в. 2, с. 315-332.

25. Mykland P. A. Asymptotic expansions for martingales. - Ann. Probab., 1993, v. 21, № 2, p. 800-818.

26. Petrov V. V. Limit Theorems of Probability Theory. Sequences of Independent Random Variables. New York: Clarendon Press, 1995, 292 p. (Oxford Stud. Probab., v. 4.)

27. Rhee $W$. An Edgeworth expansion for a sum of $m$-dependent random variables. Internat. J. Math. Math. Sci., 1985, v. 8, № 3, p. 563-569.

28. Rinott $Y$., Rotar $V$. On Edgeworth expansions for dependency-neighborhoods chain structures and Stein's method. - Probab. Theory Related Fields, 2003, v. 126, № 4, p. $528-570$.

29. Rinott $Y$., Rotar $V$. A multivariate CLT for local dependence with $n^{-1 / 2} \log n$ rate, and applications to multivariate graph related statistics. - J. Multivariate Anal., 1996 , v. 56, № 2 , p. 333-350.

30. Rotar V.I. Stein's method, Edgeworth's expansions, and a formula of Barbour. Stein's Method and Applications. Ed. by A.D. Barbour and L. H. Y. Chen. Singapore: Singapore Univ. Press, 2005, p. 59-84. (Lect. Notes Ser. Inst. Math. Sci. Natl. Univ. Singap., v. 5.)

31. Ротарь В. И. Теория вероятностей. М.: Высшая школа, 1992, 368 с.

32. Rosenblatt $M$. A central limit theorem and a strong mixing condition. - Proc. Natl. Acad. Sci. USA, 1956, v. 42, p. 43-47.

33. Статулявичус B. A. Предельные теоремы для сумм случайных величин, связанных в цепь Маркова. I, II, III. - Литов. матем. сб., 1969, т. 9, № 2, с. 345-362; 1969, т. 9, № 3, с. 635-672; 1970, т. 10, № 1, c. 161-169.

34. Stein C. A bound for the error in the normal approximation to the distribution of a sum of dependent random variables. - Proceedings of the Sixth Berkeley Symposium on Mathematical Statistics and Probability (Berkeley, 1970/1971), v. 2. Berkeley: Univ. California Press, 1972, p. 583-602.

35. Stein C. Approximate Computation of Expectations. Hayward: Inst. Math. Statist., 1986, $164 \mathrm{p}$. 